PROCEEDINGS OF THE

AMERICAN MATHEMATICAL SOCIETY

Volume 133, Number 9, Pages 2597-2604

S 0002-9939(05)08114-1

Article electronically published on April 19, 2005

\title{
ASYMPTOTIC EXPANSION OF SOLUTIONS TO NONLINEAR ELLIPTIC EIGENVALUE PROBLEMS
}

\author{
TETSUTARO SHIBATA
}

(Communicated by David S. Tartakoff)

\begin{abstract}
We consider the nonlinear eigenvalue problem
$-\Delta u+g(u)=\lambda \sin u$ in $\Omega, u>0$ in $\Omega, u=0$ on $\partial \Omega$,

where $\Omega \subset \mathbf{R}^{N}(N \geq 2)$ is an appropriately smooth bounded domain and $\lambda>0$ is a parameter. It is known that if $\lambda \gg 1$, then the corresponding solution $u_{\lambda}$ is almost flat and almost equal to $\pi$ inside $\Omega$. We establish an asymptotic expansion of $u_{\lambda}(x)(x \in \Omega)$ when $\lambda \gg 1$, which is explicitly represented by $g$.
\end{abstract}

\section{INTRODUCTION}

We consider the nonlinear eigenvalue problem

$$
\begin{aligned}
-\Delta u+g(u) & =\lambda \sin u \text { in } \Omega, \\
u & >0 \text { in } \Omega, \\
u & =0 \text { on } \partial \Omega,
\end{aligned}
$$

where $\Omega \subset \mathbf{R}^{N}(N \geq 2)$ is an appropriately smooth bounded domain and $\lambda>0$ is a parameter. We assume the following conditions (A.1)-(A.3):

(A.1) $g \in C^{m, \gamma}(\mathbf{R})(m \geq 1,0<\gamma<1)$ and $g(u)>0$ for $u>0$.

(A.2) $g(0)=g^{\prime}(0)=0$.

(A.3) $g(u) / u$ is strictly increasing for $0<u<\pi$.

The typical example of $g(u)$ is $g(u)=|u|^{m-1} u(m>1)$.

The equation (1.1)-(1.3) is regarded as the equation of a simple pendulum with a nonlinear self-interaction term $g(u)$, and the following (P.1) and (P.2) are well known and easy to show (cf. [1, 2], [4, [5]).

(P.1) For a given $\lambda \in \mathbf{R},(1.1)-(1.3)$ has a unique solution $u_{\lambda} \in C^{3}(\bar{\Omega})$ if and only if $\lambda>\lambda_{1}$, where $\lambda_{1}>0$ is the first eigenvalue of $-\Delta$ with Dirichlet zero boundary condition.

(P.2) $\left\|u_{\lambda}\right\|_{\infty}<\pi$ and $u_{\lambda} \rightarrow \pi$ locally uniformly in $\Omega$ as $\lambda \rightarrow \infty$.

Therefore, we see from (P.2) that $u_{\lambda}$ is almost flat inside $\Omega$, and one common interest to study (1.1)-(1.3) is to investigate precisely the asymptotic behavior of $u_{\lambda}$ inside $\Omega$. In other words, we are interested in "how flat $u_{\lambda}$ is inside $\Omega$ ".

In the case when $g \equiv 0$, there are many works concerning the asymptotic behavior of the solutions of (1.1)-(1.3) inside $\Omega$ as $\lambda \rightarrow \infty$, since the properties (P.1) and

Received by the editors November 7, 2003.

2000 Mathematics Subject Classification. Primary 35J60; Secondary 35P30.

Key words and phrases. Asymptotic expansion, nonlinear elliptic eigenvalue problems. 
(P.2) are valid when $g \equiv 0$ (cf. [2], [3] and the references therein). To take a simple example, let us consider the case $N=1, \Omega=(-R, R)$ and $g \equiv 0$. In this case, we denote by $u_{0, \lambda}$ the unique solution associated with given $\lambda>\lambda_{1}$. Then it is known (cf. [6]) that as $\lambda \rightarrow \infty$,

$$
\left\|u_{0, \lambda}\right\|_{\infty}=\pi-8(1+o(1)) e^{-\sqrt{\lambda}(1+o(1)) R} .
$$

We remark that the second term in the right-hand side of (1.4) decays exponentially as $\lambda \rightarrow \infty$.

However, as far as the author knows, there are no works concerning the precise asymptotic analysis of the interior behavior of $u_{\lambda}$ as $\lambda \rightarrow \infty$ when $g \not \equiv 0$. So the natural and fundamental questions we have to ask here are as follows:

(Q.1) Does the second term of $\left\|u_{\lambda}\right\|_{\infty}$ decay exponentially, too?

(Q.2) If the answer to question (Q.1) is in the negative, then what is the second term of $\left\|u_{\lambda}\right\|_{\infty}$ as $\lambda \rightarrow \infty$ ?

(Q.3) How flat is $u_{\lambda}$ inside $\Omega$ when $\lambda \gg 1$ ?

To answer these questions, we establish an asymptotic expansion of $u_{\lambda}(x)$ as $\lambda \rightarrow \infty$, which is explicitly represented by $g$ and show that the second term and the remainder terms decay algebraically as $\lambda \rightarrow \infty$.

Now we state our results. Let $G(u):=\int_{0}^{u} g(s) d s$.

Theorem 1. Let $x \in \Omega$ be fixed. Then the following asymptotic formula holds as $\lambda \rightarrow \infty$ :

$$
u_{\lambda}(x)=\pi-\sum_{k=1}^{m+1} \frac{b_{k}}{\lambda^{k}}+o\left(\frac{1}{\lambda^{m+1}}\right),
$$

where $b_{k}(k=1,2, \cdots, m+1)$ are constants determined by $\left\{g^{(j)}(\pi)\right\}_{j=0}^{k-1}$.

For example,

$$
\begin{aligned}
& b_{1}=g(\pi), \\
& b_{2}=-g(\pi) g^{\prime}(\pi), \\
& b_{3}=\frac{1}{6} g(\pi)^{3}+g(\pi)^{2} g^{\prime}(\pi)+\frac{1}{2} g(\pi)^{2} g^{(2)}(\pi) .
\end{aligned}
$$

We explain the idea of the proof of Theorem 1 briefly. We first consider (1.1)(1.3) for the case $\Omega=B_{R}:=\left\{x \in \mathbf{R}^{N}:|x|<R\right\}$. In this case, (1.1)-(1.3) are equivalent to the ordinary differential equation

$$
\begin{aligned}
& u^{\prime \prime}(r)+\frac{N-1}{r} u^{\prime}(r)-g(u(r))+\lambda \sin u(r)=0, \quad 0<r<R, \\
& u(r)>0, \quad 0 \leq r<R, \\
& u^{\prime}(0)=u(R)=0 .
\end{aligned}
$$

Then we prove (1.5) for $u_{\lambda}(0)\left(=\left\|u_{\lambda}\right\|_{\infty}\right)$. Then by using the fact that $\left\|u_{\lambda}\right\|_{\infty}$ does not depend on the radius $R$ of the ball $B_{R}$, we prove Theorem 1 . Therefore, to prove (1.5) for $\Omega=B_{R}$ and $x=0$ is crucial. The difficulty we encounter for the case $\Omega=B_{R}(N \geq 2)$ is as follows. If we directly follow the argument in [8], in which (1.5) was obtained for the case $N=1, \Omega=(-R, R)$ and $x=0$, then we find that the second term $(N-1) u_{\lambda}^{\prime}(r) / r$ in $(1.7)$, which does not appear in the case $N=1$, is quite difficult to treat. We concentrate our attention on treating this characteristic term appropriately and developing new devices to deal with the 
radial solution of (1.7)-(1.9) for the case $N \geq 2$. Then we obtain the answer to (Q.1)-(Q.3).

\section{Proof of Theorem 1 for $\Omega=B_{R}$ AND $x=0$}

In this section, we consider (1.7)-(1.9) and establish (1.5) for $x=0$. Note that $u_{\lambda}(0)=\left\|u_{\lambda}\right\|_{\infty}$. We begin with the fundamental equalities which play important roles in this section. Multiply (1.7) by $u_{\lambda}^{\prime}$. Then for $r \in[0, R]$,

$$
\left\{u_{\lambda}^{\prime \prime}(r)+\frac{N-1}{r} u_{\lambda}^{\prime}(r)+\lambda \sin u_{\lambda}(r)-g\left(u_{\lambda}(r)\right)\right\} u_{\lambda}^{\prime}(r)=0 .
$$

Since $u_{\lambda}(0)=\left\|u_{\lambda}\right\|_{\infty}$, this implies that for $r \in[0, R]$,

$$
\begin{aligned}
& \frac{1}{2} u_{\lambda}^{\prime}(r)^{2}+\int_{0}^{r} \frac{N-1}{s} u_{\lambda}^{\prime}(s)^{2} d s-\lambda \cos u_{\lambda}(r)-G\left(u_{\lambda}(r)\right) \equiv \text { constant } \\
& =-\lambda \cos \left\|u_{\lambda}\right\|_{\infty}-G\left(\left\|u_{\lambda}\right\|_{\infty}\right) \quad(\text { put } r=0) \\
& =\frac{1}{2} u_{\lambda}^{\prime}(R)^{2}+\int_{0}^{R} \frac{N-1}{s} u_{\lambda}^{\prime}(s)^{2} d s-\lambda \quad(\text { put } r=R) .
\end{aligned}
$$

Let $M_{\lambda}:=\inf \{\theta>0: \lambda \sin \theta=g(\theta)\}$. It is clear that $M_{\lambda}<\pi$ and $\lambda \sin \theta>g(\theta)$ for $0<\theta<M_{\lambda}$. We know from [1] that $\left\|u_{\lambda}\right\|_{\infty}<M_{\lambda}$. Therefore, for $0 \leq r<R$, we have

$$
\lambda \sin u_{\lambda}(r)>g\left(u_{\lambda}(r)\right) .
$$

Further, it is well known that

$$
u_{\lambda}^{\prime}(r)<0 \quad(0<r \leq R) .
$$

We begin with the following fundamental lemma.

Lemma 2.1. Let $0<r_{0}<R$ be fixed. Then $u_{\lambda}^{\prime \prime}(r)<0$ for $0 \leq r \leq r_{0}$ and $\lambda \gg 1$.

Proof. We know that for $0 \leq r \leq R$,

$$
\left(r^{N-1} u_{\lambda}^{\prime}(r)\right)^{\prime}=r^{N-1}\left(g\left(u_{\lambda}(r)\right)-\lambda \sin u_{\lambda}(r)\right) .
$$

Integrate $(2.4)$ over $[0, r]$ to obtain

$$
u_{\lambda}^{\prime}(r)=\frac{1}{r^{N-1}} \int_{0}^{r} s^{N-1}\left(g\left(u_{\lambda}(s)\right)-\lambda \sin u_{\lambda}(s)\right) d s .
$$

By this, we obtain

$$
\begin{aligned}
u_{\lambda}^{\prime \prime}(r)= & g\left(u_{\lambda}(r)\right)-\lambda \sin u_{\lambda}(r) \\
& +(N-1) r^{-N} \int_{0}^{r} s^{N-1}\left(\lambda \sin u_{\lambda}(s)-g\left(u_{\lambda}(s)\right)\right) d s .
\end{aligned}
$$

Then by integration by parts,

$$
\begin{aligned}
& \int_{0}^{r} s^{N-1}\left(\lambda \sin u_{\lambda}(s)-g\left(u_{\lambda}(s)\right)\right) d s \\
& =\frac{r^{N}}{N}\left(\lambda \sin u_{\lambda}(r)-g\left(u_{\lambda}(r)\right)\right) \\
& -\int_{0}^{r} \frac{s^{N}}{N}\left(\lambda \cos u_{\lambda}(s)-g^{\prime}\left(u_{\lambda}(s)\right)\right) u_{\lambda}^{\prime}(s) d s .
\end{aligned}
$$


By this, (P.2), (2.2), (2.3) and (2.6), for $\lambda \gg 1$, we obtain

$$
\begin{aligned}
u_{\lambda}^{\prime \prime}(r)= & -\frac{1}{N}\left(\lambda \sin u_{\lambda}(r)-g\left(u_{\lambda}(r)\right)\right) \\
& -\frac{N-1}{N} \int_{0}^{r}\left(\lambda \cos u_{\lambda}(s)-g^{\prime}\left(u_{\lambda}(s)\right)\right) u_{\lambda}^{\prime}(s) s^{N} d s \\
< & 0 .
\end{aligned}
$$

Thus the proof is complete.

Lemma 2.2. Let an arbitrary $0<r_{0}<R$ be fixed. Then $u_{\lambda}^{\prime \prime \prime}(r)<0$ for $0 \leq r \leq r_{0}$ and $\lambda \gg 1$.

Proof. By (2.6), we have

$$
\begin{aligned}
-u_{\lambda}^{\prime \prime \prime}(r)= & \left(\lambda \cos u_{\lambda}(r)-g^{\prime}\left(u_{\lambda}(r)\right)\right) u_{\lambda}^{\prime}(r) \\
& +N(N-1) r^{-N-1} \int_{0}^{r} s^{N-1}\left(\lambda \sin u_{\lambda}(s)-g\left(u_{\lambda}(s)\right)\right) d s \\
& -(N-1) r^{-1}\left(\lambda \sin u_{\lambda}(r)-g\left(u_{\lambda}(r)\right)\right) .
\end{aligned}
$$

By this and (2.7),

$$
\begin{aligned}
(2.8)-u_{\lambda}^{\prime \prime \prime}(r)= & \left(\lambda \cos u_{\lambda}(r)-g^{\prime}\left(u_{\lambda}(r)\right)\right) u_{\lambda}^{\prime}(r) \\
& -(N-1) r^{-N-1} \int_{0}^{r} s^{N}\left(\lambda \cos u_{\lambda}(s)-g^{\prime}\left(u_{\lambda}(s)\right)\right) u_{\lambda}^{\prime}(s) d s .
\end{aligned}
$$

Since $\lambda \gg 1$, by (P.2) and integration by parts, we obtain

$$
\begin{aligned}
& \int_{0}^{r} s^{N}\left(\lambda \cos u_{\lambda}(s)-g^{\prime}\left(u_{\lambda}(s)\right)\right) u_{\lambda}^{\prime}(s) d s \\
& =-\left(\lambda+g^{\prime}(\pi)\right)(1+o(1)) \int_{0}^{r} s^{N} u_{\lambda}^{\prime}(s) d s \\
& =-\left(\lambda+g^{\prime}(\pi)\right)(1+o(1))\left(\frac{1}{N+1} r^{N+1} u_{\lambda}^{\prime}(r)-\int_{0}^{r} \frac{1}{N+1} s^{N+1} u_{\lambda}^{\prime \prime}(s) d s\right) .
\end{aligned}
$$

By this, Lemma 2.1, (2.3) and (2.8), for $0 \leq r \leq r_{0}$ and $\lambda \gg 1$, we obtain

$$
\begin{aligned}
(2.9)-u_{\lambda}^{\prime \prime \prime}(r)= & \frac{2}{N+1}\left(\lambda+g^{\prime}(\pi)\right)(1+o(1))\left(-u_{\lambda}^{\prime}(r)\right) \\
& +\frac{N-1}{N+1} r^{-N-1}\left(\lambda+g^{\prime}(\pi)\right)(1+o(1)) \int_{0}^{r} s^{N+1}\left(-u_{\lambda}^{\prime \prime}(s)\right) d s \\
> & 0 .
\end{aligned}
$$

Thus the proof is complete.

We put

$$
\xi_{\lambda}:=\lambda \sin \left\|u_{\lambda}\right\|_{\infty}-g\left(\left\|u_{\lambda}\right\|_{\infty}\right)
$$

By l'Hopital's rule,

$$
\lim _{r \rightarrow 0} \frac{u_{\lambda}^{\prime}(r)}{r}=\lim _{r \rightarrow 0} u_{\lambda}^{\prime \prime}(r)=u_{\lambda}^{\prime \prime}(0) .
$$

By this and (1.7), we obtain

$$
N u_{\lambda}^{\prime \prime}(0)+\lambda \sin \left\|u_{\lambda}\right\|_{\infty}-g\left(\left\|u_{\lambda}\right\|_{\infty}\right)=0 .
$$


This along with (2.10) and Lemma 2.1 implies that

$$
\xi_{\lambda}=-N u_{\lambda}^{\prime \prime}(0)>0 .
$$

Furthermore, we put

$$
\begin{aligned}
I_{\lambda}(r) & :=\lambda\left(\cos u_{\lambda}(r)-\cos \left\|u_{\lambda}\right\|_{\infty}\right)+G\left(u_{\lambda}(r)\right)-G\left(\left\|u_{\lambda}\right\|_{\infty}\right), \\
I I_{\lambda}(r) & :=\int_{0}^{r} \frac{N-1}{s} u_{\lambda}^{\prime}(s)^{2} d s .
\end{aligned}
$$

Then for $r \in[0, R]$, by $(2.1)$, we obtain

$$
\frac{1}{2} u_{\lambda}^{\prime}(r)^{2}=I_{\lambda}(r)-I I_{\lambda}(r)
$$

Lemma 2.3. Let $0<r_{0}<R$ be fixed. Then for $0 \leq r \leq r_{0}$ and $\lambda \gg 1$

$$
I_{\lambda}(r)=\xi_{\lambda}\left(\left\|u_{\lambda}\right\|_{\infty}-u_{\lambda}(r)\right)+\frac{1}{2}\left(\lambda+g^{\prime}(\pi)\right)(1+o(1))\left(\left\|u_{\lambda}\right\|_{\infty}-u_{\lambda}(r)\right)^{2} .
$$

Proof. By Taylor expansion and (P.2), for $0 \leq r \leq r_{0}$ and $\lambda \gg 1$, we obtain

$$
\begin{aligned}
\cos u_{\lambda}(r)= & \cos \left\|u_{\lambda}\right\|_{\infty}-\sin \left\|u_{\lambda}\right\|_{\infty}\left(u_{\lambda}(r)-\left\|u_{\lambda}\right\|_{\infty}\right) \\
& -\frac{1}{2} \cos \left\|u_{\lambda}\right\|_{\infty}\left(u_{\lambda}(r)-\left\|u_{\lambda}\right\|_{\infty}\right)^{2}(1+o(1)), \\
G\left(u_{\lambda}(r)\right)= & G\left(\left\|u_{\lambda}\right\|_{\infty}\right)+g\left(\left\|u_{\lambda}\right\|_{\infty}\right)\left(u_{\lambda}(r)-\left\|u_{\lambda}\right\|_{\infty}\right) \\
& +\frac{1}{2}\left(g^{\prime}\left(\left\|u_{\lambda}\right\|_{\infty}\right)+o(1)\right)\left(u_{\lambda}(r)-\left\|u_{\lambda}\right\|_{\infty}\right)^{2} .
\end{aligned}
$$

Then by (P.2), (2.12), (2.16) and (2.17), for $0 \leq r \leq r_{0}$ and $\lambda \gg 1$, we have

$$
\begin{aligned}
I_{\lambda} & =\lambda\left(\cos u_{\lambda}(r)-\cos \left\|u_{\lambda}\right\|_{\infty}\right)+G\left(u_{\lambda}(r)\right)-G\left(\left\|u_{\lambda}\right\|_{\infty}\right) \\
& =\xi_{\lambda}\left(\left\|u_{\lambda}\right\|_{\infty}-u_{\lambda}(r)\right)+\frac{1}{2}\left(\lambda+g^{\prime}(\pi)\right)(1+o(1))\left(\left\|u_{\lambda}\right\|_{\infty}-u_{\lambda}(r)\right)^{2} .
\end{aligned}
$$

Lemma 2.4. Let $0<r_{0}<R$ be fixed. Then for $0 \leq r \leq r_{0}$ and $\lambda \gg 1$,

$$
\begin{aligned}
I I_{\lambda}(r) \leq & \frac{N-1}{N} \xi_{\lambda}\left(\left\|u_{\lambda}\right\|_{\infty}-u_{\lambda}(r)\right) \\
& +\frac{N-1}{2(N+1)}\left(\lambda+g^{\prime}(\pi)\right)(1+o(1))\left(\left\|u_{\lambda}\right\|_{\infty}-u_{\lambda}(r)\right)^{2} .
\end{aligned}
$$

Proof. Since (2.18) is valid for $r=0$, let $r>0$. By l'Hopital's rule and (2.11),

$$
\begin{aligned}
\lim _{r \rightarrow 0} \frac{I I_{\lambda}(r)}{\left\|u_{\lambda}\right\|_{\infty}-u_{\lambda}(r)} & =\lim _{r \rightarrow 0} \frac{-(N-1) u_{\lambda}^{\prime}(r)}{r}=\lim _{r \rightarrow 0}(1-N) u_{\lambda}^{\prime \prime}(r) \\
& =(1-N) u_{\lambda}^{\prime \prime}(0)=\frac{N-1}{N} \xi_{\lambda} .
\end{aligned}
$$


Then by this and Cauchy's mean value theorem, for $0<r \leq r_{0}$ and $\lambda \gg 1$, we obtain

$$
\begin{aligned}
& \frac{I I_{\lambda}(r)-\left((N-1) \xi_{\lambda} / N\right)\left(\left\|u_{\lambda}\right\|_{\infty}-u_{\lambda}(r)\right)}{\left(\left\|u_{\lambda}\right\|_{\infty}-u_{\lambda}(r)\right)^{2}} \\
& =\frac{(N-1) u_{\lambda}^{\prime}\left(r_{1}\right)+(N-1) \xi_{\lambda} r_{1} / N}{-2 r_{1}\left(\left\|u_{\lambda}\right\|_{\infty}-u_{\lambda}\left(r_{1}\right)\right)} \\
& =\frac{(N-1) u_{\lambda}^{\prime \prime}\left(r_{2}\right)+(N-1) \xi_{\lambda} / N}{-2\left(\left\|u_{\lambda}\right\|_{\infty}-u_{\lambda}\left(r_{2}\right)\right)+2 r_{2} u_{\lambda}^{\prime}\left(r_{2}\right)} \\
& =\frac{(N-1) u_{\lambda}^{\prime \prime \prime}\left(r_{3}\right)}{4 u_{\lambda}^{\prime}\left(r_{3}\right)+2 r_{3} u_{\lambda}^{\prime \prime}\left(r_{3}\right)}
\end{aligned}
$$

where $0<r_{3}<r_{2}<r_{1}<r$. By Lemma 2.2, we see that $-u_{\lambda}^{\prime \prime}(r)$ is increasing for $0 \leq r \leq r_{0}$. Then by (2.9), for any $0 \leq r \leq r_{0}$ and $\lambda \gg 1$, we obtain

$$
\begin{aligned}
-u_{\lambda}^{\prime \prime \prime}(r) \leq & \frac{2}{N+2}\left(\lambda+g^{\prime}(\pi)\right)(1+o(1))\left(-u_{\lambda}^{\prime}(r)\right) \\
& +\frac{N-1}{N+1} r^{-N-1}\left(\lambda+g^{\prime}(\pi)\right)(1+o(1))\left(-u_{\lambda}^{\prime \prime}(r)\right) \int_{0}^{r} s^{N+1} d s \\
\leq & \frac{2}{N+2}\left(\lambda+g^{\prime}(\pi)\right)(1+o(1))\left(-u_{\lambda}^{\prime}(r)\right) \\
& +\frac{N-1}{(N+1)(N+2)}\left(\lambda+g^{\prime}(\pi)\right)(1+o(1)) r\left(-u_{\lambda}^{\prime \prime}(r)\right) \\
\leq & \frac{1}{2(N+1)}\left(\lambda+g^{\prime}(\pi)\right)(1+o(1))\left(-4 u_{\lambda}^{\prime}(r)-2 r u_{\lambda}^{\prime \prime}(r)\right) .
\end{aligned}
$$

Put $r=r_{3}$ in (2.21). Then by this and (2.20), we obtain (2.18). Thus the proof is complete.

Lemma 2.5. Let $0<r_{0}<R$ be fixed. Then $\xi_{\lambda}=o\left(\lambda e^{-\sqrt{2 \lambda(1+o(1)) /(N+1)} r_{0}}\right)$ as $\lambda \rightarrow \infty$.

Proof. By (2.14), Lemmas 2.3 and 2.4, for $0 \leq r \leq r_{0}$ and $\lambda \gg 1$,

$$
\begin{aligned}
\frac{1}{2} u_{\lambda}^{\prime}(r)^{2}= & I_{\lambda}(r)-I I_{\lambda}(r) \\
\geq & \frac{1}{N} \xi_{\lambda}\left(\left\|u_{\lambda}\right\|_{\infty}-u_{\lambda}(r)\right) \\
& +\frac{1}{N+1}(1+o(1))\left(g^{\prime}(\pi)+\lambda\right)\left(\left\|u_{\lambda}\right\|_{\infty}-u_{\lambda}(r)\right)^{2} \\
\geq & \frac{1}{2} P_{\lambda}\left(\left\|u_{\lambda}\right\|_{\infty}-u_{\lambda}(r)\right)+\frac{1}{2} Q_{\lambda}\left(\left\|u_{\lambda}\right\|_{\infty}-u_{\lambda}(r)\right)^{2},
\end{aligned}
$$


where $P_{\lambda}:=2 \xi_{\lambda} / N, Q_{\lambda}:=2 \lambda(1+o(1)) /(N+1)$. We put $t=\sqrt{s /\left(P_{\lambda}+Q_{\lambda} s\right)}$. Then by (2.14) and (2.22), for $\lambda \gg 1$, we obtain

$$
\begin{aligned}
r_{0} & =\int_{0}^{r_{0}} 1 d t=\int_{0}^{r_{0}} \frac{-u_{\lambda}^{\prime}(r)}{\sqrt{2\left(I_{\lambda}(r)-I I_{\lambda}(r)\right)}} d r \\
& \leq \int_{u_{\lambda}\left(r_{0}\right)}^{\left\|u_{\lambda}\right\|_{\infty}} \frac{1}{\sqrt{P_{\lambda}\left(\left\|u_{\lambda}\right\|_{\infty}-\theta\right)+Q_{\lambda}\left(\left\|u_{\lambda}\right\|_{\infty}-\theta\right)^{2}}} d \theta \\
& =\int_{0}^{\left\|u_{\lambda}\right\|_{\infty}-u_{\lambda}\left(r_{0}\right)} \frac{1}{\sqrt{P_{\lambda} s+Q_{\lambda} s^{2}}} d s=\int_{0}^{A_{\lambda}} \frac{2}{1-Q_{\lambda} t^{2}} d t \\
& =\frac{1}{\sqrt{Q_{\lambda}}} \log \left(\frac{1 / \sqrt{Q_{\lambda}}+A_{\lambda}}{1 / \sqrt{Q_{\lambda}}-A_{\lambda}}\right),
\end{aligned}
$$

where

$$
A_{\lambda}:=\sqrt{\frac{\left\|u_{\lambda}\right\|_{\infty}-u_{\lambda}\left(r_{0}\right)}{P_{\lambda}+Q_{\lambda}\left(\left\|u_{\lambda}\right\|_{\infty}-u_{\lambda}\left(r_{0}\right)\right)}} .
$$

This implies that

$$
\sqrt{\frac{\left(\left\|u_{\lambda}\right\|_{\infty}-u_{\lambda}\left(r_{0}\right)\right)}{P_{\lambda}+Q_{\lambda}\left(\left\|u_{\lambda}\right\|_{\infty}-u_{\lambda}\left(r_{0}\right)\right)}} \geq \frac{1}{\sqrt{Q_{\lambda}}} \frac{e^{\sqrt{Q_{\lambda}} r_{0}}-1}{e^{\sqrt{Q_{\lambda}} r_{0}}+1} .
$$

By this and (P.2), for $\lambda \gg 1$, we obtain

$$
\frac{2}{N} \xi_{\lambda}=P_{\lambda} \leq \frac{4 Q_{\lambda} \lambda e^{\sqrt{Q_{\lambda}} r_{0}}\left(\left\|u_{\lambda}\right\|_{\infty}-u_{\lambda}\left(r_{0}\right)\right)}{\left(e^{\sqrt{Q_{\lambda}} r_{0}}-1\right)^{2}}=o\left(\lambda e^{-\sqrt{2 \lambda(1+o(1)) /(N+1)} r_{0}}\right) .
$$

Thus the proof is complete.

Proof of Theorem 1 for $\Omega=B_{R}$ and $x=0$. We put $\zeta_{1}(\lambda):=\pi-\left\|u_{\lambda}\right\|_{\infty}$. Then $\zeta_{1}(\lambda) \rightarrow 0$ as $\lambda \rightarrow \infty$ by (P.2). Then by Lemma 2.5 , we obtain

$$
\begin{aligned}
\lambda \sin \left\|u_{\lambda}\right\|_{\infty} & =\lambda \sin \left(\pi-\zeta_{1}(\lambda)\right)=\lambda \sin \zeta_{1}(\lambda) \\
& =\lambda(1+o(1)) \zeta_{1}(\lambda) \\
& =g\left(\left\|u_{\lambda}\right\|_{\infty}\right)+o\left(\lambda e^{-\sqrt{2 \lambda(1+o(1) /(N+1)} r_{0}}\right) \\
& =g(\pi)(1+o(1)) .
\end{aligned}
$$

This implies that

$$
\zeta_{1}(\lambda)=\frac{g(\pi)}{\lambda}+o\left(\frac{1}{\lambda}\right) .
$$

Therefore, we obtain the second term in (1.5). Then (1.5) is proved by completely the same argument as that in [7. Proof of Theorem 2], which is proceeded by the mathematical induction. Thus the proof of Theorem 1 for the case $\Omega=B_{R}$ and $x=0$ is complete.

\section{Proof of Theorem 1}

Now we consider (1.1)-(1.3). Let $x \in \Omega$ be fixed. Let $\delta_{1}:=\operatorname{dist}\{x, \partial \Omega\}>0$ and $L_{1}:=\sup \left\{\left|y_{1}-y_{2}\right|: y_{1}, y_{2} \in \Omega\right\}$. Since (1.1)-(1.3) is autonomous, by translation of the coordinate system, we may assume that $x=0$. Let $B_{1}=B_{\delta_{1} / 2}$ and $B_{2}:=B_{2 L_{1}}$. Furthermore, let $u_{\lambda, 1}$ and $u_{\lambda, 2}$ be the solutions of (1.7)-(1.9) for $R=\delta_{1} / 2$ and $R=2 L_{1}$, respectively. It is clear that $u_{\lambda}$ is a super-solution of (1.1)-(1.3) for $B_{1}$. 
Further, for $0<\epsilon \ll 1$, we see from (A.2) that $v_{\epsilon}(|x|)=\epsilon \varphi_{1}(|x|)$ is a sub-solution of (1.1)-(1.3) for $B_{1}$, where $\varphi_{1}$ is the first positive eigenfunction of $-\Delta$ in $B_{1}$ with the Dirichlet zero boundary condition. Since $0<\epsilon \ll 1$, we see that $\epsilon \varphi_{1}<u_{\lambda}$. Then since $u_{\lambda, 1}$ is a unique solution of (1.1)-(1.3) for $\Omega=B_{1}$, we see from [7, p. $24]$ that for $x \in B_{1}$

$$
\epsilon \varphi_{1}(x) \leq u_{\lambda, 1}(x) \leq u_{\lambda}(x) .
$$

By the same argument as above, for $x \in \Omega$, we have

$$
u_{\lambda}(x) \leq u_{\lambda, 2}(x) .
$$

In particular, by putting $x=0$, we obtain

$$
\left\|u_{\lambda, 1}\right\|_{\infty} \leq u_{\lambda}(0) \leq\left\|u_{\lambda, 2}\right\|_{\infty} .
$$

Since the formula (1.5) holds for $\left\|u_{\lambda, 1}\right\|_{\infty}$ and $\left\|u_{\lambda, 2}\right\|_{\infty}$, by (3.2) and (3.3), we immediately obtain (1.5). Thus the proof is complete.

\section{REFERENCES}

[1] P. Clément and G. Sweers, Existence and multiplicity results for a semilinear elliptic eigenvalue problem, Ann. Scuola Norm. Sup. Pisa Cl. Sci. (4) 14 (1987), 97-121. MR0937538 (89j:35053)

[2] F. A. Howes, Singularly perturbed semilinear elliptic boundary value problems, Comm. in Partial Differential Equations 4 (1979), 1-39. MF0514718 (81i:35072)

[3] F. A. Howes, Boundary-interior layer interactions in nonlinear singular perturbation theory, Mem. Amer. Math. Soc. 15, No. 203 (1978). MR0499407 (58:17288)

[4] R. E. O'Malley, Jr., Phase-plane solutions to some singular perturbation problems, J. Math. Anal. Appl. 54 (1976), 449-466. MR0450722 (56:9015)

[5] R. E. O'Malley, Jr., "Singular perturbation methods for ordinary differential equations", Springer, New York, 1989. MR1123483 (92i:34071)

[6] T. Shibata, Precise spectral asymptotics for the Dirichlet problem $-u^{\prime \prime}(t)+g(u(t))=\lambda \sin u(t)$, J. Math. Anal. Appl. 267 (2002), 576-598. MR1888025 (2003b:34048)

[7] D. H. Sattinger, "Topics in stability and bifurcation theory", Lect. Notes in Math. 309, Springer, New York, 1973. MR0463624 (57:3569)

[8] T. Shibata, Asymptotic expansion of the boundary layers of the perturbed simple pendulum problems, J. Math. Anal. Appl. 283 (2003), 431-439. MR1991818 (2004b:34141)

Department of Applied Mathematics, Graduate School of Engineering, Hiroshima University, Higashi-Hiroshima, 739-8527, JaPAN

E-mail address: shibata@amath.hiroshima-u.ac.jp 